\title{
FABRICATION OF 3D PARYLENE SHEATH PROBES FOR RELIABLE NEUROPROSTHETIC RECORDINGS
}

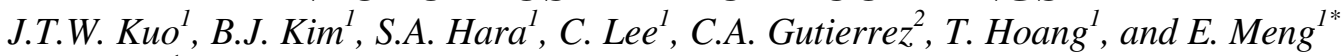 \\ ${ }^{1}$ University of Southern California, Los Angeles, California, USA \\ ${ }^{2}$ Los Angeles, California, USA
}

\begin{abstract}
3D Parylene sheath probes having Pt electrodes on the outer and inner surfaces of the sheath are introduced as a novel interface for long term intracortical neural recording. Surface micromachined Parylene channels with Pt electrodes are expanded into a $3 \mathrm{D}$ sheath structure by thermoforming in the presence of a custom tapered microwire that shapes the sheath. Electrochemical characterization, including electrochemical impedance spectroscopy (EIS) and cyclic voltammetry (CV), of the Pt electrodes sites was performed. EIS yielded electrode impedances ranging from $20-60 \mathrm{k} \Omega$ at $1 \mathrm{kHz}$. Electrode characterization results suggest the thermoforming process does not impact electrode performance and were in agreement with characteristics of typical neural recording electrodes. These sheath probes with their 3D structural design, soft biocompatible materials, and bioactive coatings promote integration with neural tissue enabling reliable, long term cortical recordings.
\end{abstract}

\section{INTRODUCTION}

Brain-machine interfaces for controlling motor prostheses require reliable chronic recordings from intracortical microelectrodes. Current microelectrode technologies are hampered by signal degradation over time that are attributed in part to neurotoxic factors released by microglia due to inflammatory response arising from the mechanical mismatch between the implanted rigid probe and cortical tissue; this leads to gradual retraction of neural processes from recording sites [1].

Intracortical microelectrode technologies possess short lifetime in terms of acceptable recording quality ( $<5$ years). There are two such microelectrode formats that have been used in clinical studies. The first was constructed of tapered-tip silicon pins with a single electrode at the tip [2-5]. Thermomigration was used to create trails of $\mathrm{p}^{+}$type silicon through a wafer which were exposed by removal of n-type silicon to create arrays of needles that are subsequently coated with platinum. Glass cone electrodes in which the cones are manually fabricated from pulled pipettes have achieved the most reliable long term recordings in human to date [6-12]. Microwires with de-insulated tips placed into the cone interior at different depths serve as the recording sites, with 2-4 wires per cone. Living sciatic nerve tissue or neurotrophic factors were placed within the cones prior to implantation. The ingrowth of dendritic processes into the cone toward cortical recording sites ( $\sim 3$ months) were attributed to the release of neurotrophic factors. Although, long-term recording reliability was achieved, manual fabrication of the device is labor intensive and not amenable to manufacturability nor increase in the number of recording sites.

Improvement in long term intracortical recordings is necessary to realize practical neural prostheses. Critical considerations in neural interface design include selection of appropriate biocompatible structural materials to alleviate the mechanical mismatch with tissue. Repeatability across devices and batch fabrication can be achieved by using microfabrication technologies. The used of thin film microfabricated electrodes also increases the number and density of recording sites per probe, while minimizing footprint, in order to acquire greater spatiotemporal signal information compared to microwire approaches. Finally, the use of appropriate bioactive coatings can reduce inflammatory response and promote integration into tissue.

To achieve a stable long-term intracortical interface, we introduce a novel microfabricated structure consisting of a hollow sheath in the form of a cone or cylinder that can be decorated with multiple biologically functional coatings (NGF, collagen, Matrigel, etc.) to mitigate the inflammatory response and attract and maintain growth of neural processes toward recording sites (Figure 1). Openings at the ends of the sheath further facilitate ingrowth of neural processes after recovery from implantation toward recording sites and tissue integration.
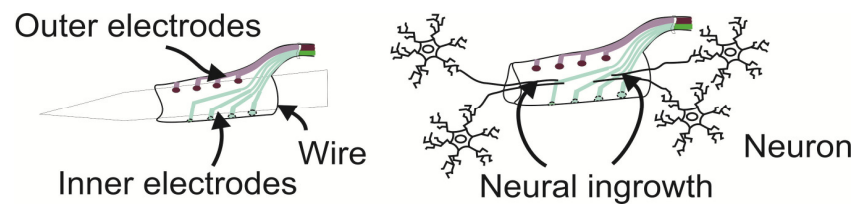

Figure 1: Conceptual drawing of Parylene sheath probe for long term intracortical recordings. A wire is inserted into the sheath and used as a surgical introducer to implant the device into the cortex (left). The wire is then removed and ingrowth of neural processes following release of bioactive coatings facilitates long term stable recordings (right).

\section{METHODS}

\section{Material Selection}

Parylene C, a Class VI biocompatible polymer that can be processed with standard micromachining technologies [13], was selected as the sheath probe structural material. The use of Parylene $\mathrm{C}$ mitigates mechanical mismatch issues between rigid probe and soft tissue present in previous technologies. Parylene $\mathrm{C}$ is flexible and much more pliable than stiff materials such as $\mathrm{Si}$ and glass possessing a low Young's modulus [14].

Only Pt was used for electrodes since no adhesion layer is needed with a Parylene substrate [15]. Pt is also highly biocompatible and widely used in bioMEMS neural interface applications [16-17].

\section{Device Design}

Sheath probes are cone or cylinder shaped with $4 \mathrm{Pt}$ electrodes (45 $\mu \mathrm{m}$ diameter) on each of the inner and outer surfaces. An integrated flexible Parylene cable $(\sim 2 \mathrm{~cm}$ long) is included. Sheath dimensions and electrode placement were selected to match the neural anatomy of the barrel cortex in rat for eventual neural recordings to capture vibrissal (whisker) movements.

The barrel cortex is organized into distinct regions that map to individual vibrissae. Individual vibrissae movements are mediated by neurons in layers $\mathrm{V}$ and VI of the barrel cortex through generation of action potentials. These layers are located at a depth of $\sim 1.5-2.0 \mathrm{~mm}$ in the rat brain and span $\sim 800 \mu \mathrm{m}$ in height [18]. Thus, the sheath structure was designed to be $800 \mu \mathrm{m}$ in length to span the targeted pyramidal layers. Electrodes were spaced equidistant from each other within the sheath length in order to effectively capture neural signals spanning the two layers. 


\section{Fabrication}

A silicon substrate was used for mechanical support during the fabrication process (Figure 2). A $5 \mu \mathrm{m}$ Parylene layer was first deposited onto the substrate to serve as the base layer of the device. Inner sheath e-beam deposited Pt electrodes $(2000 \AA)$ were then patterned by a liftoff process with negative photoresist $\mathrm{AZ}$ 5214 E-IR. These electrodes will record from dendritic processes extending into the sheath. A $1 \mu \mathrm{m}$ Parylene insulation layer was then deposited and selectively plasma etched to expose electrodes and contact pads. Sacrificial photoresist AZ 4620 was spun to a height of $8 \mu \mathrm{m}$ and patterned to form the sheath structures. A $5 \mu \mathrm{m}$ Parylene layer was then deposited over the photoresist.

Outer electrodes were patterned on top of the sheath structure using a dual layer liftoff scheme. AZ 1518 was first spun on and globally exposed. AZ 4620 was then spun on and patterned with the top electrodes. This dual photoresist AZ 1518/AZ 4620 layer scheme produced a negative sidewall profile that enhances the liftoff process for thicker photoresists and was necessary to ensure a continuous pattern from the top of the structure to the base [19]. As with inner electrodes, Pt was e-beam deposited (2000 $⿱$ A) for outer electrodes. A final $1 \mu \mathrm{m}$ Parylene insulation layer was deposited and plasma etched to create openings for outer electrodes and contact pads. Plasma etching then created sheath openings and the device cutout. Sacrificial photoresist was removed with an acetone soak and the device was released from the substrate.
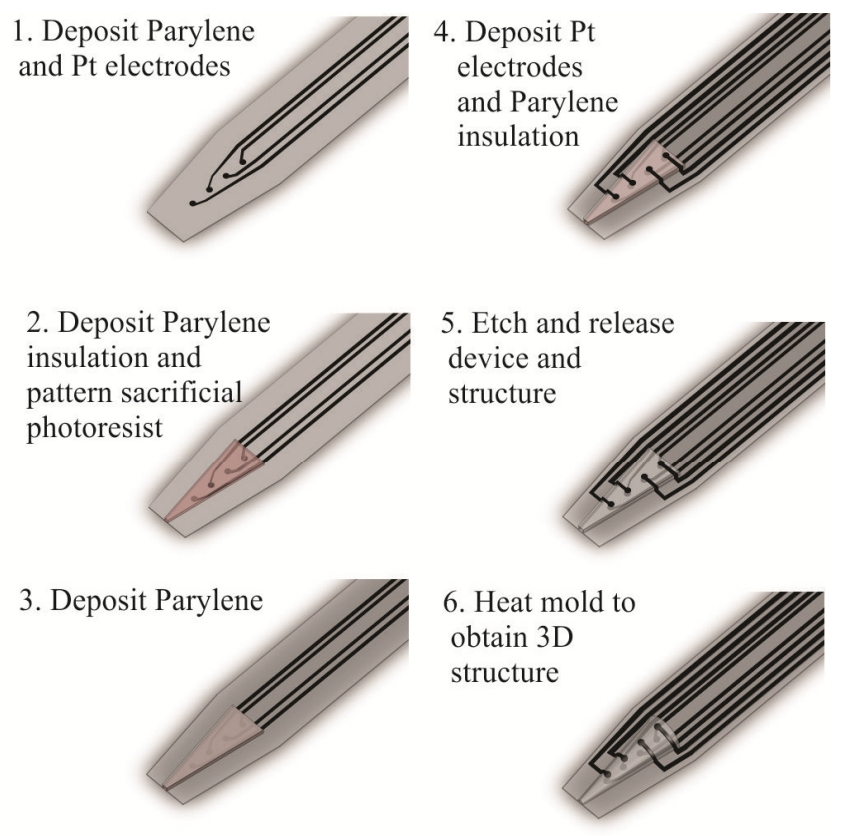

Figure 2: Abbreviated fabrication process which utilizes standard surface micromachining techniques followed by thermoforming. Final outline of device is shown throughout process for clarity.

\section{Parylene Thermoforming}

A custom tapered stainless steel or tungsten microwire was used as a mold for thermoforming of the conical or cylindrical structure (Figure 3). Microwires were etched to match the desired probe shape and supplied by MicroProbes for Life Science. The shaped microwire tip was carefully inserted into the sheath underneath a microscope. The wire and device were placed in a vacuum oven and the Parylene sheath was thermoformed by controlled temperature ramp up to $200{ }^{\circ} \mathrm{C}$ for 48 hours with nitrogen purging. After thermoforming, the wire was removed and the sheath retained its 3D shape (Figure 4).

Thermoforming not only provided the desired 3D shape but also annealed the Parylene layers [20]. This improves the adhesion between Parylene layers. Just prior to implantation, the sheaths are coated with neurotrophic factors. A zero insertion force (ZIF) connector was used to establish reversible electrical connections to the microelectrodes of the sheath through the contact pads at the end of the integrated Parylene ribbon cable (Figure 5) [21].

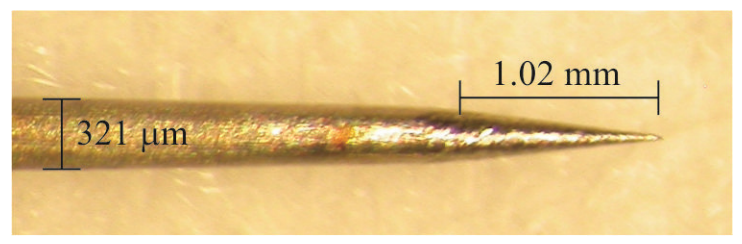

Figure 3: Custom tapered stainless steel wire for insertion into sheath for thermoforming.

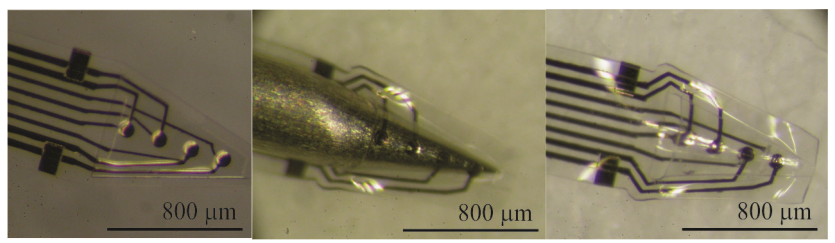

Figure 4: The sacrificial layer was first released (left).The tapered wire was inserted into the sheath (middle) and the structure was thermoformed. After thermoforming and removal of the wire, the sheath held its $3 D$ shape (right).

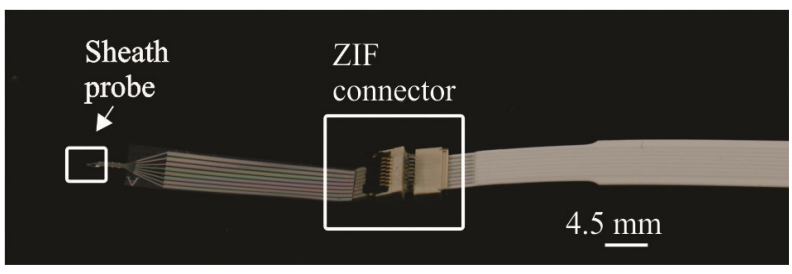

Figure 5: Sheath probe with integrated Parylene cable is attached to a ZIF connector for external electrical connection.

\section{RESULTS \\ 3D Sheath Probe Fabrication}

Sheath probes were successfully fabricated with integrated Parylene ribbon cables (Figure 6). In total, five different design variants were fabricated for eventual use in rat whisker intracortical recordings through the barrel cortex (Table 1).

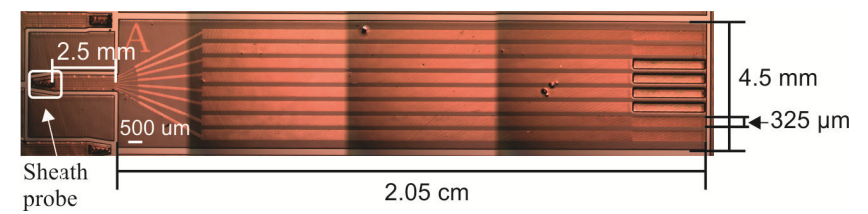

Figure 6: Photograph showing layout of unreleased probe with integrated cable still covered with photoresist. The vertical artifacts appearing along the length of the ribbon cable are due to stitching of images to obtain a view of the full length of the probe. 
Electrodes were placed to match the targeted cortical layers. 4 different cone shapes with different tapers ending at a $50 \mu \mathrm{m}$ opening were fabricated in order to test the effect of different geometries on dendritic ingrowth, if any (Figure 7). A cylinder structure was also fabricated.

Table 1: Dimensions of the different fabricated sheath structures. Electrode placement and dimensions remained constant for all device variants.

\begin{tabular}{|l|c|c|c|}
\hline $\begin{array}{l}\text { Device } \\
\text { Nomenclature }\end{array}$ & $\begin{array}{c}\text { Base Opening } \\
\text { Diameter }\end{array}$ & $\begin{array}{c}\text { Tip Opening } \\
\text { Diameter }\end{array}$ & $\begin{array}{c}\text { Sheath } \\
\text { Length }\end{array}$ \\
\hline A & $300 \mu \mathrm{m}$ & $50 \mu \mathrm{m}$ & $800 \mu \mathrm{m}$ \\
\hline $\mathrm{B}$ & $450 \mu \mathrm{m}$ & $50 \mu \mathrm{m}$ & $800 \mu \mathrm{m}$ \\
\hline $\mathrm{C}$ & $300 \mu \mathrm{m}$ & $300 \mu \mathrm{m}$ & $800 \mu \mathrm{m}$ \\
\hline A-T & $500 \mu \mathrm{m}$ & $50 \mu \mathrm{m}$ & $800 \mu \mathrm{m}$ \\
\hline B-T & $650 \mu \mathrm{m}$ & $50 \mu \mathrm{m}$ & $800 \mu \mathrm{m}$ \\
\hline
\end{tabular}
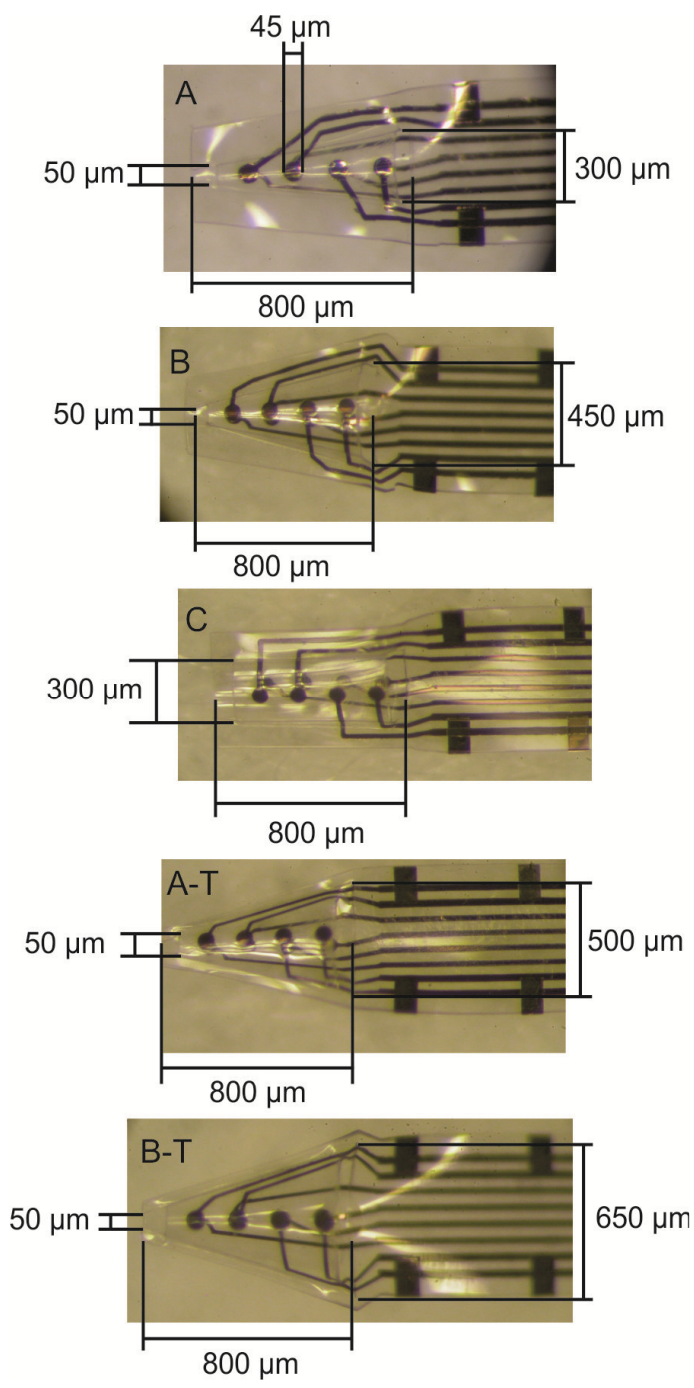

Figure 7: Photographs of the different sheath designs. Each device has been thermoformed. Electrode placement matches rat barrel cortex anatomy.

Thermoformed sheaths were mechanically robust and unlike microelectrodes supported on thin Parylene films, could be directly implanted into agarose $(0.5 \%)$ models of neural tissue without additional mechanical support.

\section{Outer Sheath Surface Electrodes}

In all designs, fabrication of the outer surface electrodes was achieved successfully with the dual photoresist layer liftoff method despite the nonplanar nature of the structure. Visual inspection through microscopy showed no discontinuity between the lead traces from the top of the structure to the bottom. Thermoformed devices exhibited no discontinuities in leads to the outer surface electrodes despite the increased strain.

A microprobe attached to a micromanipulator was carefully positioned over an electrode on the outer cone surface while another was positioned over the corresponding contact pad on the Parylene cable. A Keithley 2700 MultiMeter was used to measure the lead resistance which was observed to range from 325-389 $\Omega$ across all leads. Conductivity between the electrode and contact pad confirmed electrical continuity as leads transition from the bottom to the top of the sheath.

\section{Cyclic Voltammetry}

Cyclic voltammetry (CV) of Pt electrodes was conducted on a Gamry Reference 600 potentiostat (Figure 8). The sheath probe was placed in a $0.05 \mathrm{M}$ solution of sulfuric acid $\left(\mathrm{H}_{2} \mathrm{SO}_{4}\right)$ with one of the $\mathrm{Pt}$ electrodes used as the working electrode. $\mathrm{An} \mathrm{Ag} \mathrm{Ag} / \mathrm{Cl}$ electrode in a $3 \mathrm{M} \mathrm{NaCl}$ solution was used as a reference electrode. An adjacent Pt electrode was used as the counter electrode. A scan rate of $250 \mathrm{mV} / \mathrm{sec}$ was used and cycled from -0.2 to $1.2 \mathrm{~V}$ until stable voltammograms were achieved. The resulting stable voltammogram was recorded and the procedure was repeated for the remaining Pt electrodes.

The standard voltammogram for Pt immersed in sulfuric acid was achieved for all electrodes. Oxide reduction and oxidation peaks were clearly observed as well as the hydrogen peaks indicating desorption and adsorption from the Pt surface. Thus, electrical connections from the ZIF connector to the contact pad through the Parylene cables to the recording site was verified to be electrically conductive. Micromachined thin film Pt was validated to possess the same properties as a bulk Pt microwire and so can be expected to yield similar neural recordings.

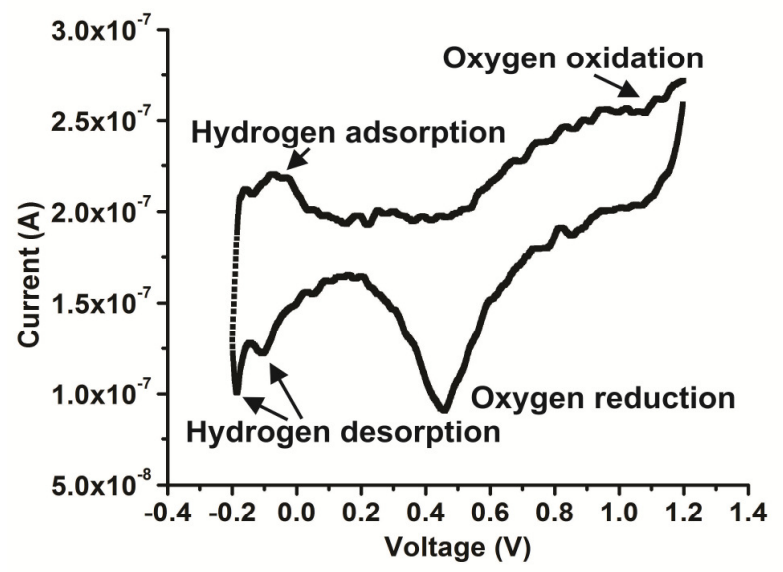

Figure 8: $\mathrm{CV}$ in $\mathrm{H}_{2} \mathrm{SO}_{4}$ of single recording site yielded standard $\mathrm{Pt}$ electrode characteristics.

Electrochemical impedance spectroscopy (EIS) was 
performed on the electrodes with the sheath probe immersed in $1 \mathrm{X}$ phosphate buffer solution (PBS). A representative measurement for a single electrode site is shown in Figure 9.

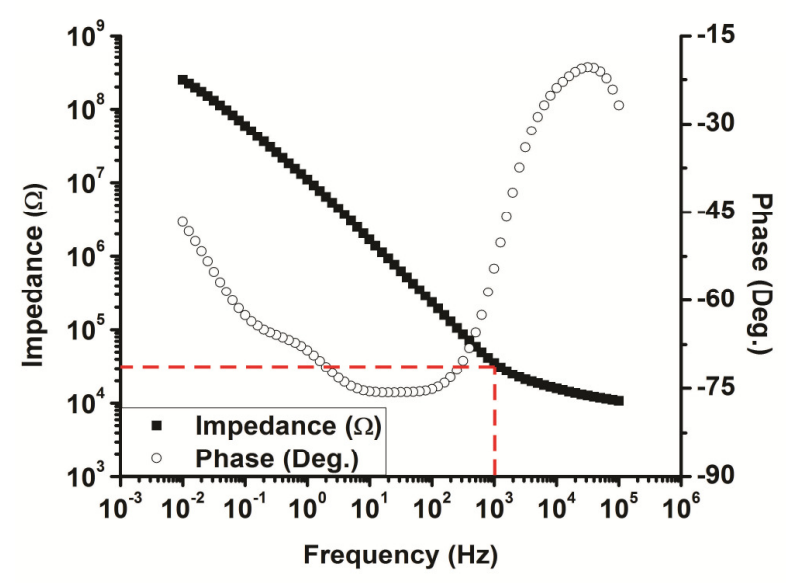

Figure 9: EIS of a single Pt recording electrode immersed in $1 X$ $P B S$. Impedance is $31.25 \mathrm{k} \Omega$ at $1 \mathrm{kHz}$.

As before, a standard phase and impedance plot were observed for all electrodes indicating that the thin film $\mathrm{Pt}$ electrodes are expected to behave in a similar fashion to $\mathrm{Pt}$ microwires for neural recordings. Recorded impedances were found to range from $20-60 \mathrm{k} \Omega$ at $1 \mathrm{kHz}$ are expected to provide adequate neural recording potential. It is desirable to limit the electrode impedance to achieve high SNR due to neural signal attenuation from the voltage divider created by electrode impedance and input impedance of recording equipment [22].

\section{CONCLUSION}

We introduced a novel 3D Parylene sheath probe that was micromachined and then thermoformed for intracortical neural recordings. Compared to earlier glass cone electrodes, this process was able to produce devices having repeatable dimensions in a batch process. Microfabrication also enabled greater yield compared to manually made cone electrodes. Parylene $\mathrm{C}$ was selected as the structural material for its biocompatibility and improved mechanical matching to tissue compared to rigid $\mathrm{Si}$ and glass.

Sheath structures were fabricated with electrodes at the outer and inner surfaces. Sheath dimensions and electrode placement were designed to match rat barrel cortex neuroanatomy. Prior to implantation in the brain, these hollow 3D structures will be coated with neurotrophic factors and immunosuppressants to promote dendritic ingrowth and reduce inflammatory response.

Electrical connectivity to probes was achieved using a ZIF connection method. Recording electrodes were characterized with $\mathrm{CV}$ and EIS. Results indicated that thermoforming did not adversely impact electrochemical behavior of the electrodes.

Future work entails evaluation of the efficacy of long term cortical recordings from the probes implanted in rat barrel cortex.

\section{ACKNOWLEDGEMENTS}

The authors wish to thank Dr. Donghai Zhu and members of the Biomedical Microsystems Lab for their assistance.

This work was sponsored by the Defense Advanced Research Projects Agency (DARPA) MTO under the auspices of Dr. Jack
Judy through the Space and Naval Warfare Systems Center, Pacific Grant/Contract No. N66001-11-1-4207.

\section{REFERENCES}

1. V. S. Polikov, P. A. Tresco, W. M. Reichert, J. Neurosci. Methods 148, 1 (2005).

2. P. K. Campbell, K. E. Jones, R. J. Huber, K. W. Horch, R. A. Normann, Biomedical Engineering, IEEE Transactions on 38, 758 (1991).

3. L. R. Hochberg et al., Nature 442, 164 (Jul 13, 2006).

4. E. M. Maynard, C. T. Nordhausen, R. A. Normann, Electroencephalogr Clin Neurophysiol 102, 228 (Mar, 1997).

5. S. Suner, M. R. Fellows, C. Vargas-Irwin, G. K. Nakata, J. P. Donoghue, IEEE Trans Neural Syst Rehabil Eng 13, 524 (Dec, 2005).

6. J. Bartels et al., Journal of Neuroscience Methods 174, 168 (Sep 30, 2008).

7. F. H. Guenther et al., Plos One 4 (Dec 9, 2009).

8. P. Kennedy et al., Journal of neural engineering 1, 72 (2004).

9. P. R. Kennedy, J. Neurosci. Methods 29, 181 (1989).

10. P. R. Kennedy, R. A. Bakay, Neuroreport 9, 1707 (Jun 1, 1998).

11. P. R. Kennedy, R. A. Bakay, S. M. Sharpe, Neuroreport: An International Journal for the Rapid Communication of Research in Neuroscience; Neuroreport: An International Journal for the Rapid Communication of Research in Neuroscience (1992).

12. P. R. Kennedy, S. S. Mirra, R. A. E. Bakay, Neuroscience letters 142, 89 (1992).

13. E. Meng, X. Zhang, W. Benard, in MEMS Materials and Processes Handbook R. Ghodssi, P. Li, Eds. (Springer, 2011) pp. 193-271.

14. T. A. Harder, Y. Tze-Jung, H. Qing, S. Chi-Yuan, T. YuChong, in The 15th IEEE International Conference on Micro Electro Mechanical Systems, MEMS'02. (2002) pp. 435-438.

15. D. C. Rodger et al., Sensors and Actuators B: Chemical 132, 449 (2008).

16. E. Meng, Biomedical Microsystems (CRC Press, ed. 1, 2010), pp. 408.

17. J. T. W. Kuo, L.-Y. Chang, P.-Y. Li, T. Hoang, E. Meng, Sensors and Actuators B: Chemical 152, 267 (2011).

18. M. Brecht, M. Schneider, B. Sakmann, T. W. Margrie, Nature 427, 704 (2004).

19. C. Gutierrez, E. Meng, in Transducers 2009. (IEEE, Denver, Colorado, USA, , 2009) pp. 2194-2197.

20. B. J. Kim, B. Chen, E. Meng, in Submitted to Hilton Head 2012: A Solid State Sensors, Actuators and Microsystems Workshop. (IEEE, 2012).

21. C. A. Gutierrez, C. Lee, B. Kim, E. Meng, in 16th International Solid-State Sensors, Actuators and Microsystems Conference (TRANSDUCERS). (IEEE, Beijing, China, 5-9 June 2011) pp. 2299-2302.

22. D. A. Borkholder, Stanford University (1998).

\section{CONTACT}

*E. Meng, tel: +1-213-821-3949; ellis.meng@usc.edu 